\title{
EL USO DE LA FAUNA EN LAGUNA CÓNDOR, PROVINCIA DE SANTA CRUZ, ARGENTINA
}

\author{
G. LORENA L'HEUREUX'a \& LUIS A. BORREROa
}

\begin{abstract}
RESUMEN
El presente trabajo expone los resultados del análisis de la fauna recuperada en la localidad arqueológica de Laguna Cóndor, ubicada en el curso superior del río Gallegos en la zona de los Morros, Santa Cruz, Argentina. A pesar de la baja tasa de depositación ósea general, se registraron elevadas densidades puntuales de huesos muy fragmentados. Se identificó una alta diversidad de especies recuperadas aunque un único taxón fue explotado en forma intensa. El uso de la fauna estuvo dirigido fundamentalmente a la explotación de Lama guanicoe y, en forma ocasional, a recursos de bosque (Hippocamelus bisulcus) y a fauna introducida (Equus caballus). La evidencia refuerza la interpretación de Laguna Cóndor como un espacio explotado logísticamente en forma intensa y recurrente, pero por periodos relativamente breves hacia ca. 200 años AP.
\end{abstract}

PALABRAS CLAVES: laguna Cóndor, uso de la fauna, Holoceno tardío.

\section{FAUNAL EXPLOITATION AT LAGUNA CONDOR SITE, SANTA CRUZ PROVINCE, ARGENTINA}

\begin{abstract}
This paper presents the results of the analysis of faunal assemblages recovered at the archaeological locality of Laguna Condor, on the upper basin of the Gallegos River at the "Morros area", Santa Cruz, Argentina. Despite the low rate of bone deposition, high densities of highly fragmented bones were recorded. A high diversity of species was identified, although only one taxon was intensively exploited. The focus was on the exploitation of Lama guanicoe and, occasionally, forest resources (Hippocamelus bisulcus), and European fauna (Equus caballus). The evidence reinforces the interpretation of Laguna Condor as logistically, redundantly and intensively exploited place during short periods ca. 200 years AP.
\end{abstract}

KEY WORDS: laguna Condor site, fauna use, late Holocene.

a IMHICIHU-CONICET. Saavedra 15 (ACA 1083), Ciudad de Buenos Aires, Argentina. lorenalheureux@gmail.com; laborrero2003@yahoo.com 


\section{INTRODUCCIÓN}

El objetivo del presente trabajo es presentar y discutir los resultados del análisis faunístico de la localidad arqueológica Laguna Cóndor, localizada dentro de los límites de la actual Estancia Glencross en la margen Norte del río Turbio, cuenca superior del río Gallegos (Provincia de Santa Cruz, Argentina; Fig. 1). El sitio Laguna

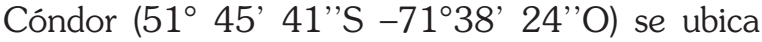
en un área de ecotono bosque-estepa, que también es denominada de "Los Morros", en alusión a los puntos más elevados del paisaje (400 msnm): el morro Phillippi (el más próximo a Laguna Cóndor), el Domeyko y el Gay (Fig. 1).

Hasta el primer lustro del siglo XXI, los antecedentes arqueológicos del área postulaban un uso poco intenso del sector de la cuenca superior del río Gallegos. La evidencia arqueológica, aunque escasa y discontinua, demostraba la explotación de este espacio por parte de los cazadores recolectores desde, por lo menos, comienzos del Holoceno tardío (Carballo Marina et al. 2008; Gómez Otero, 1991; Molinari, 2000; Ortiz Troncoso, 1973). La distribución de hallazgos arqueológicos planteaba como límite occidental de uso y explotación aborigen el sector comprendido por la Laguna Cóndor y el Morro Philippi y el morro era considerado el sitio más occidental en las nacientes del río Gallegos (Gómez Otero, 1991).

Partiendo de los antecedentes arriba mencionados, en 2008 se retomaron las investigaciones en el sector occidental de la Laguna Cóndor, la cual había sido caracterizada como una "zona vacía" de información arqueológica (Borrero et al. 2008). Los estudios sistemáticos demostraron, en contraposición con la hipótesis de la zona vacía, que existió un uso recurrente del área, aunque se confirmó que este nunca fue intenso. Se identificaron numerosas concentraciones de hallazgos líticos en la Laguna Cóndor, en la zona de ecotono y al occidente de la misma, ya en zona de bosque (Charlin et al. 2011). La nueva evidencia cambió el conocimiento arqueológico del área que, previamente, señalaba una baja intensidad de hallazgos distribuidos espacialmente en forma discontinua solo hacia el oriente de la Laguna Cóndor (Carballo Marina et al. 2008; Gómez Otero, 1991). Igualmente, se mantuvo la noción de una extremadamente baja intensidad de uso en la zona intermedia entre Pali Aike y el este de Baguales.

En el marco de lo expuesto, este trabajo presenta el análisis de la fauna recuperada en la localidad arqueológica de Laguna Cóndor. Se pretende aportar información faunística novedosa, que sea de utilidad para completar el rol que tuvo este sector del espacio en la subsistencia de los cazadores recolectores que habitaron el área durante el Holoceno tardío.

\section{LA LAGUNA CÓNDOR}

La laguna es un cuerpo de agua permanente de origen glaciar que posee un perímetro aproximado de $14.806 \mathrm{~m}$. En su margen Norte conserva un parche de bosque mixto relictual.

El relicto de bosque se compone de ñire (Nothofagus antarctica) y lenga (Nothofagus pumilio), siendo el ñire el Nothofagus con mayor variación morfológica y mayor tolerancia ecológica. Su plasticidad fenotípica ha facilitado la conservación del bosque en cotas bajas, el cual se vio afectado por la desforestación, la desertificación, la ganadería ovina y la explotación carbonífera en áreas adyacentes. El morfotipo del bosque observado en la margen de la laguna es del tipo arbóreo-arbustivo y arbustivo achaparrado, característico de espacios semiabiertos expuestos a los intensos vientos del Oeste que dominan la región (Peri et al. 2013; Ramírez et al. 1985).

\section{El Sitio Laguna Cóndor}

En el sitio Laguna Cóndor (LC) se excavaron cuatro sondeos de $50 \mathrm{~cm}^{2}$, tres próximos a la costa Norte de la laguna (sondeos 1, 2 y 3) y otro dentro de la cárcava de un médano deflacionado (sondeo 4) ubicado a $150 \mathrm{~m}$ de la costa (Fig. 1). Aproximadamente a $300 \mathrm{~m}$ de los sondeos se realizó un muestreo de $3 \mathrm{~m}^{2}$ en un basural en el que se recogieron restos de fauna además de fragmentos de vidrio, botellas de gres y piezas de metal que permitieron ubicarlo temporalmente hacia fines del siglo XIX (Buscaglia, 2011). Un segundo muestreo se planteó sobre una concentración de materiales identificada en el sector de médanos de la margen norte de la laguna. 
a

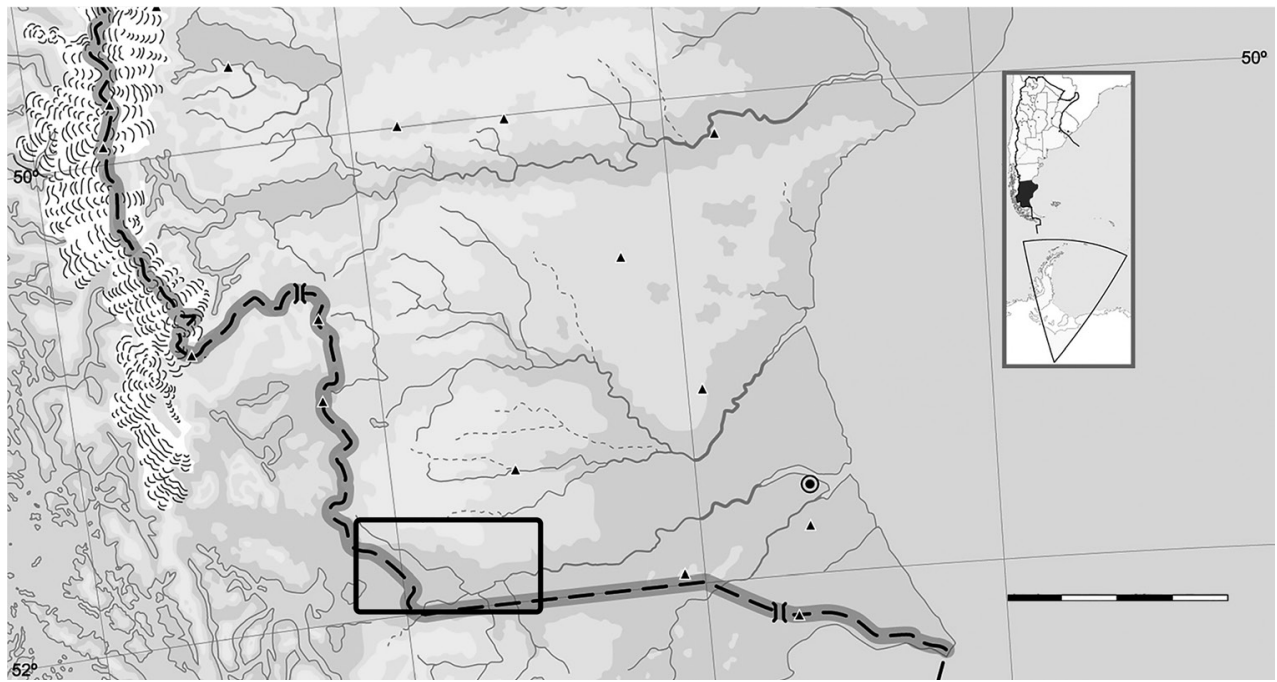

b

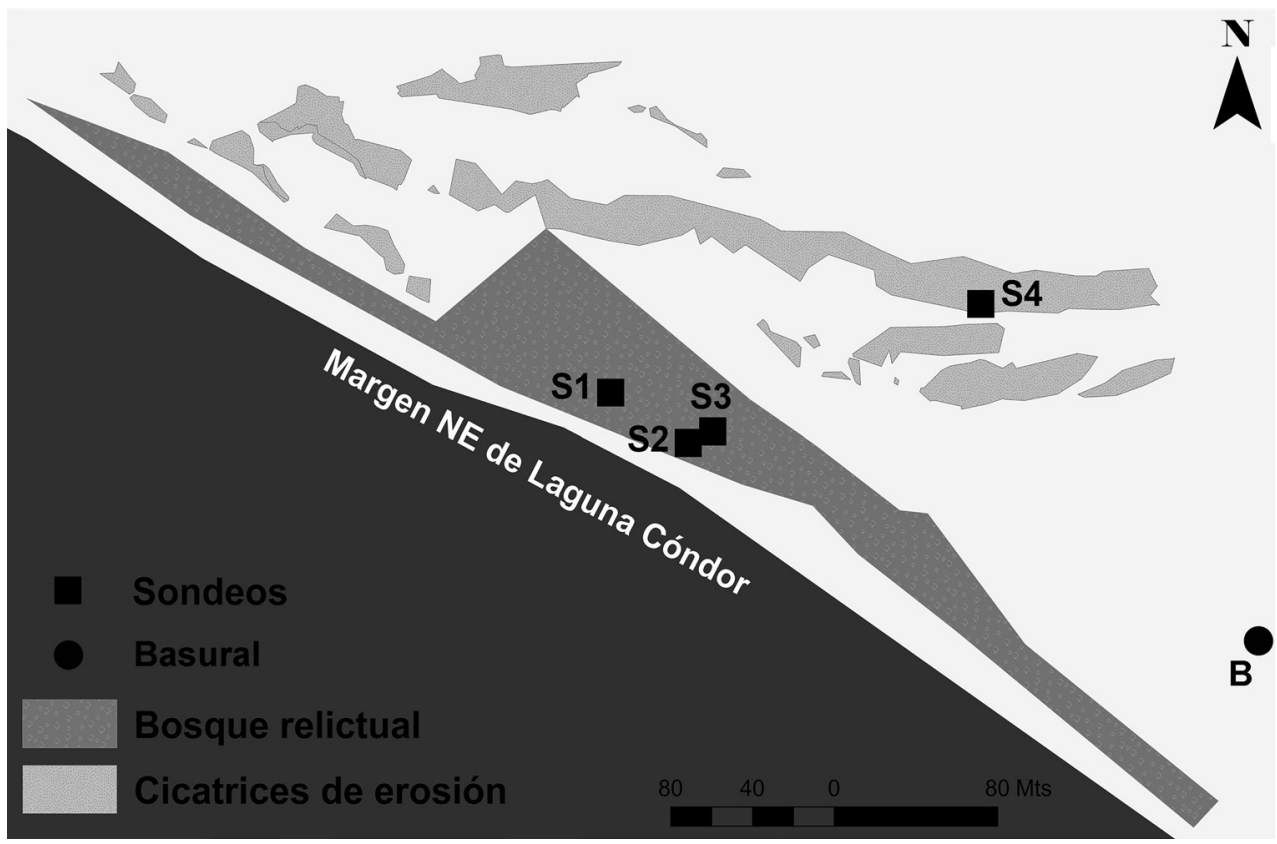

Fig. 1. (a) Cuenca superior del Río Gallegos; (b) Ubicación del sector de bosque, las cicatrices de erosión y los sondeos realizados en la margen Norte de la Laguna Cóndor.

En este trabajo se analiza la fauna recuperada en los sondeos mencionados, los restos óseos hallados en el perfil del médano erosionado, y el material correspondiente a las recolecciones superficiales de los muestreos de la margen Norte de la laguna y del sector denominado basurero histórico (Fig. 1).

El sondeo 1 se localiza sobre el perfil de un médano próximo a la laguna. Se excavaron niveles artificiales de $10 \mathrm{~cm}$ hasta alcanzar 117 $\mathrm{cm}$ de profundidad, luego se profundizó a pala hasta la base $(133 \mathrm{~cm})$ donde el sedimento, arenoso y suelto, cambia hacia uno limo-arcilloso más compacto. El sondeo 2 se planteó sobre un perfil de escasa potencia en el borde de la laguna, debajo de un calafate donde se había registrado una concentración de carbones, artefactos líticos y huesos. El sedimento arenoso se excavó mediante niveles artificiales de $5 \mathrm{~cm}$ llegando a un nivel estéril a los $35 \mathrm{~cm}$ de profundidad. El 
Tabla 1. Fechados radiocarbónicos disponibles para la localidad de Laguna cóndor.

\begin{tabular}{llllll}
\hline AA \# & Material & Procedencia & ${ }^{14} \mathrm{C} \mathrm{AP}$ & $\delta^{13} \mathrm{C}$ & Referencia \\
\hline AA91422 & guanaco & Médano $(68 \mathrm{~cm})$ & $220 \pm 41$ & -21.0 & Charlin et al. 2011 \\
AA91423 & guanaco & Médano $(107 \mathrm{~cm})$ & $187 \pm 41$ & -20.8 & Charlin et al. 2011 \\
AA98668 & guanaco & S1 $(47-57 \mathrm{~cm})$ & $213 \pm 43$ & -20.8 & este trabajo \\
AA98669 & guanaco & S3 $(18-28 \mathrm{~cm})$ & $232 \pm 43$ & -21.3 & este trabajo \\
\hline
\end{tabular}

sondeo 3, localizado en el perfil de la barranca en el borde de la laguna, se excavó por niveles artificiales de $10 \mathrm{~cm}$ hasta la base a $60 \mathrm{~cm}$ de profundidad. El sedimento arenoso cambió a uno arcilloso y compacto hacia los $50 \mathrm{~cm}$ de profundidad donde dejaron de observarse los hallazgos. El sondeo 4 se realizó sobre una concentración de huesos de guanaco localizada en la cárcava de un médano deflacionado. Se excavó un único nivel de $20 \mathrm{~cm}$ sobre el piso del cañadón que, en ese lugar, era de unos 95 $\mathrm{cm}$ profundidad, y donde se observaba el límite de la formación de un suelo que presentaba una potencia de entre 2 y $6 \mathrm{~cm}$. Los huesos se hallaron dentro del suelo.

Las dataciones radiocarbónicas disponibles para Laguna Cóndor ubican temporalmente la ocupación del sitio en momentos históricos (Tabla 1). Las edades obtenidas en los distintos loci son congruentes y señalan la ocupación del área para momentos comprendidos dentro de la denominada Pequeña Edad de Hielo.

\section{RESULTADOS}

En total, en Laguna Cóndor (LC) se recuperaron 330 especímenes de fauna ${ }^{1}$ (Tabla 2). En el conjunto se registraron especies nativas e introducidas, silvestres y domésticas, destacándose en estratigrafía el predominio del guanaco seguido por restos de mamíferos indeterminados, y una mayor variedad de taxa en superficie (Tabla 2). La mezcla de fauna de origen europeo y especies con rastros de explotación aborigen caracteriza los conjuntos de superficie. Un elemento de oveja, hallado entre los 18 y $28 \mathrm{~cm}$ de profundidad del sondeo 3, evidencia asimismo el potencial de

1 Los especímenes intrusivos de tuco tuco distribuidos entre los 30 y $60 \mathrm{~cm}$ del sondeo 3 y la concentración natural de caracoles terrestres hallada en superficie en proximidades
Tabla 2. Frecuencias de especímenes óseos, elementos e individuos identificados en Laguna Cóndor. $\mathrm{NISP}=$ número de especímenes. $\mathrm{MNE}=$ número mínimo de elementos. $\mathrm{MNI}=$ número mínimo de individuos.

\begin{tabular}{|c|c|c|c|c|}
\hline UNIDAD & TAXA & NISP & MNE & MNI \\
\hline SONDEO 1 & Lama guanicoe & 15 & 2 & 1 \\
\hline \multirow{2}{*}{ SONDEO 2} & Mammalia & 1 & 1 & 1 \\
\hline & Lepus europaeus & 1 & 1 & 1 \\
\hline \multirow{6}{*}{ SONDEO 3} & Lama guanicoe & 74 & 12 & 1 \\
\hline & Ovis aries & 1 & 1 & 1 \\
\hline & Mammalia & 74 & 3 & 1 \\
\hline & Nycticorax $n$. & 1 & 1 & 1 \\
\hline & Ave & 11 & 4 & 1 \\
\hline & Ctenomys sp.* & 40 & 22 & 3 \\
\hline SONDEO 4 & Lama guanicoe & 3 & 3 & 1 \\
\hline MÉDANO & Lama guanicoe & 12 & 9 & 1 \\
\hline \multirow{7}{*}{$\begin{array}{l}\text { SUP. } \\
\text { MARGEN N }\end{array}$} & Lama guanicoe & 27 & 14 & 1 \\
\hline & Hippocamelus $b$. & 1 & 1 & 1 \\
\hline & Equus f. caballus & 1 & 1 & 1 \\
\hline & Lepus europaeus & 1 & 1 & 1 \\
\hline & Ctenomys sp. & 21 & 20 & 2 \\
\hline & Ave & 1 & 1 & 1 \\
\hline & Gasteropoda* & 23 & 23 & 23 \\
\hline \multirow{6}{*}{$\begin{array}{l}\text { SUP. } \\
\text { BASURERO }\end{array}$} & Hippocamelus b. & 3 & 3 & 1 \\
\hline & Artiodactyla & 8 & 7 & 1 \\
\hline & Ovis aries & 1 & 1 & 1 \\
\hline & Equus f. caballus & 2 & 2 & 1 \\
\hline & Puma concolor & 2 & 2 & 1 \\
\hline & Mammalia & 6 & 3 & 1 \\
\hline
\end{tabular}

*No se incluyen en los análisis subsiguientes.

del sondeo 4, no fueron considerados en los análisis subsiguientes. 
Fig. 2. (a) Riqueza de especies en Laguna Cóndor (95\% de confidencia); (b) Riqueza de especies por unidad de estudio (95\% de confidencia).
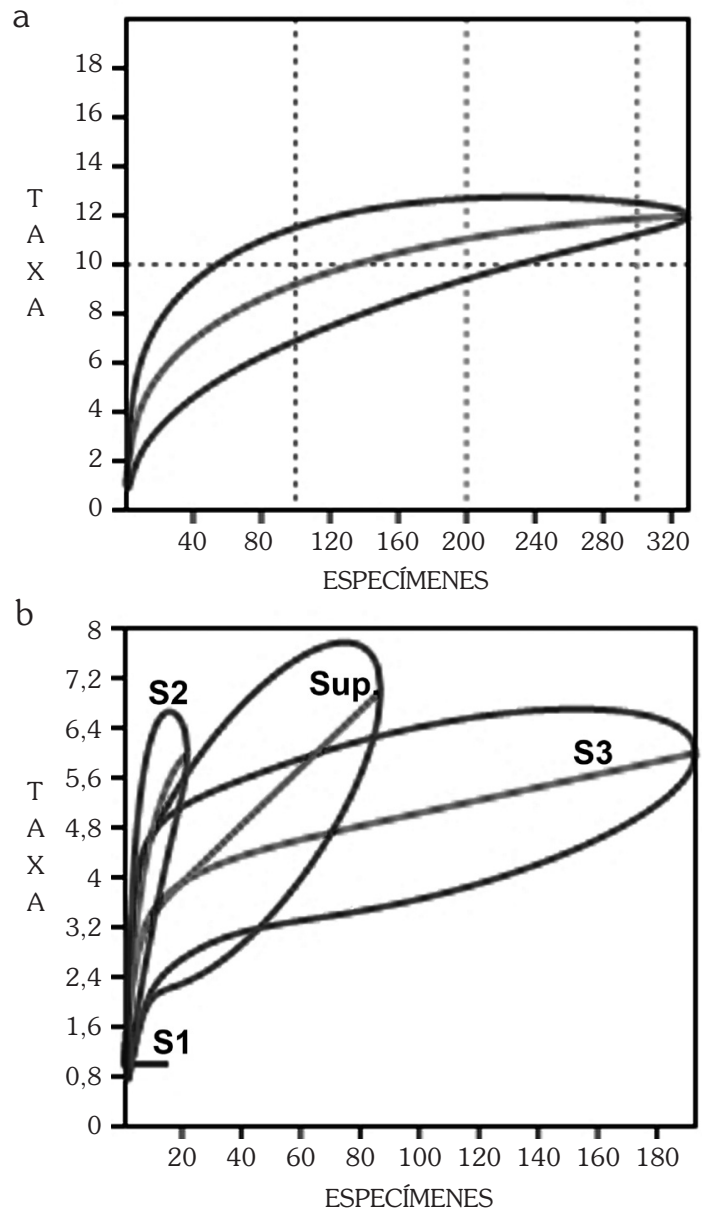

mezcla de material arqueológico y moderno del depósito.

En los muestreos las clases identificadas incrementan notablemente respecto de lo observado en las excavaciones (Tabla 2), llamando la atención la presencia de puma y de huemul (ver infra). La diferencia en la diversidad de especies representadas (riqueza) se evaluó mediante curvas de rarefacción, un método sensible a las especies raras y libre de problemas de muestreo (Gotelli \& Graves, 1996), por lo que resultó viable para aplicar sobre las muestras pequeñas de LC.

$\mathrm{Al}$ analizar la fauna del sitio en conjunto, las curvas de rarefacción muestran una elevada variedad de taxa presentes en el sitio, ya sea, evaluada por NISP como por MNI (Fig. 2a). Pero, al considerar las diferentes unidades de análisis, el análisis de rarefacción muestra otro panorama. La segmentación redujo la riqueza de cada muestra, siendo notablemente mayor para el sondeo 3 (la curva de rarefacción alcanza la forma asintótica). En los sondeos 1, 2, 4 y en

Fig. 3. (a) Estadios de meteorización; (b) Variables tafonómicas registradas sobre los especímenes óseos; (c) Variables tafonómicas antrópicas registradas en LC.

a

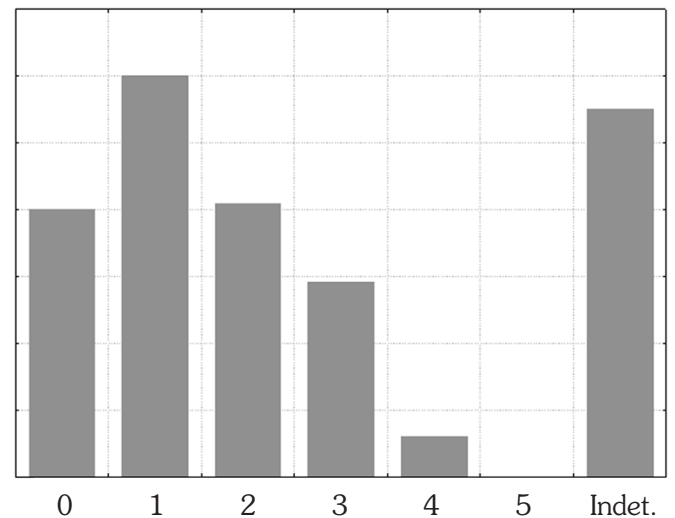

$\mathrm{b}$

ROEDORES

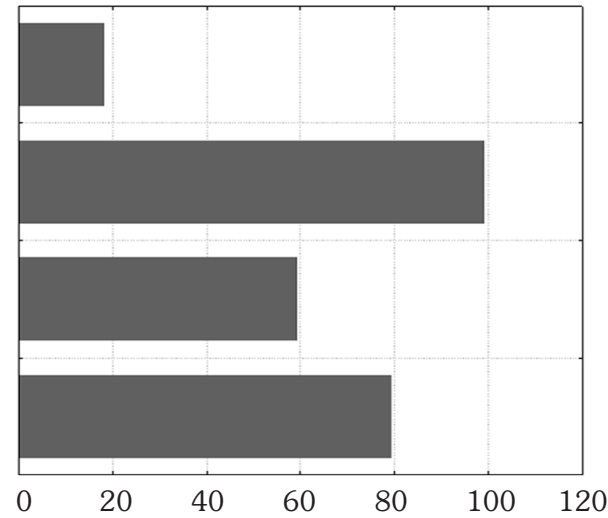

MANGANESO

EROSIÓN

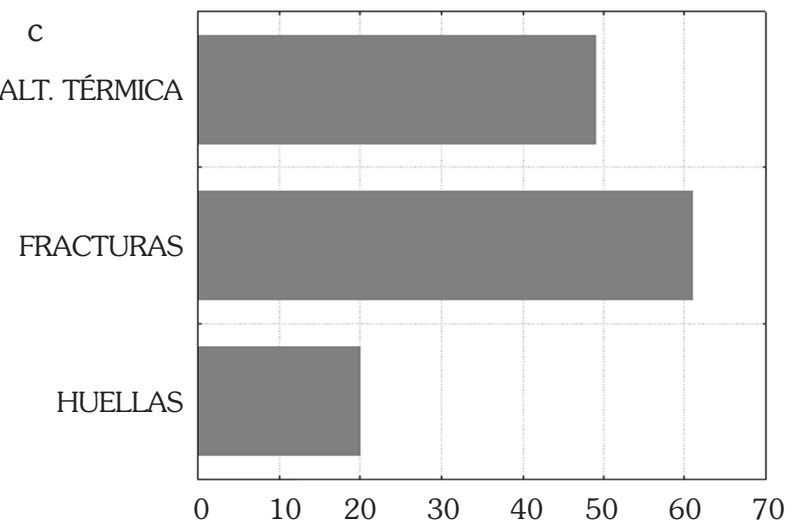


el médano, la abundancia relativa resulta heterogénea (la curva incrementa lentamente en forma lineal). Esto sería reflejo de la dominancia de guanaco en dichas unidades de análisis (Fig. 2b).

La muestra de mamíferos de LC presentó un perfil de meteorización (sensu Behrensmeyer, 1978) bajo a moderado (Fig. 3a). Predominan los elementos con estadio 1 (25,97 \%), seguidos de restos con estadios 0 y $2(17,75$ $\%$ y $17,32 \%$ respectivamente). No se registraron diferencias entre los conjuntos de estratigrafía y superficie, aunque en este último grupo incrementan significativamente los especímenes con estadios de meteorización indeterminada.

Las modificaciones naturales registradas con mayor frecuencia fueron la acción de raíces $(32,25 \%)$ y la erosión de las superficies óseas (25,73\%). En menor proporción se presentó la tinción con $\mathrm{MnO}_{2}$ $(19,47 \%) y$, en baja frecuencia, la acción de roedores $(8,14 \%)$ (Fig. 3b). Más del $61 \%$ de las modificaciones naturales se registraron sobre los especímenes de guanaco, determinando que el patrón observado para el sitio en general sea similar al registrado en este taxón. Es de notar que, a pesar de registrarse la presencia en el sitio de una de las especies de carnivoro que habita en el área, no se observaron marcas de carnivoro sobre los restos óseos.

En relación con las modificaciones de naturaleza antrópica, en el conjunto de la fauna se observaron 20 especímenes con huellas de corte y/o raspado (Fig. 3c), de las cuales el $90 \%$ se registran sobre elementos de guanaco y una sobre un espécimen de huemul (primera falange entera hallada en superficie). Las fracturas de naturaleza antrópica $(n=61)$ se registraron fundamentalmente sobre elementos de guanaco (78,69 \%), pero también sobre restos de Mammalia (11,48\%), de Equus caballus y Artiodactyla (4,92 \% para cada taxón).
Fig. 4. (a) Tamaños (mm) de los especímenes en Laguna Cóndor; (b) Índice de fragmentación de Lama guanicoe.

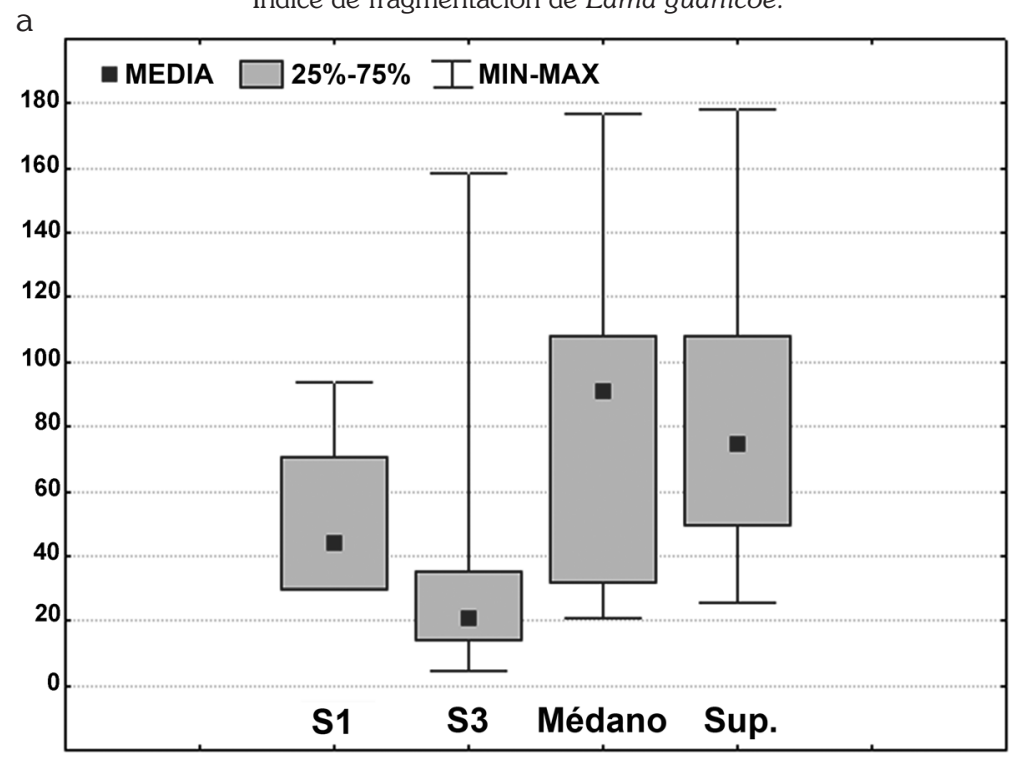

\begin{tabular}{lccc}
\hline Lama guanicoe & NISP & MNE & ÍNDICE FRAG. \\
\hline Sondeo 1 & 15 & 2 & 7.50 \\
Sondeo 3 & 74 & 12 & 6.17 \\
Sondeo 4 & 3 & 3 & 1.00 \\
Superficie & 27 & 14 & 1.93 \\
Médano & 12 & 9 & 1.33 \\
\hline Laguna Cóndor & 131 & 40 & 3.28 \\
\hline
\end{tabular}

La alteración térmica afectó el 21,60 \% de los especímenes de LC ( $\mathrm{n}=49)$. El carbonizado y calcinado en los huesos de guanaco se asocia con otras modificaciones de procesamiento $(14,29 \%$; Fig. 3c). La totalidad de los elementos alterados térmicamente se hallaron en el sondeo 3, con excepción dos restos de guanaco recuperados en el sondeo 4 y dos de caballo procedentes del muestreo en la margen Norte de la laguna.

Los tamaños de los especímenes óseos registrados en los sondeos 1 y 3 (Fig. 4a) y el elevado índice de fragmentación registrado en los restos de guanaco (Fig. 4b), sustentan la idea de un uso intenso de los recursos en Laguna Cóndor hacia momentos históricos.

La principal presa procesada en el sitio fue el guanaco (Figs. 5a y 6a). Si se consideran todas las unidades de agregación, en el sitio se explotaron por lo menos 5 individuos (MNI=5; Lyman, 1994). Las clases de edad representadas, de acuerdo con 
Fig. 5. (a) Splinter de radiocúbito derecho de Lama guanicoe hallado a $40 \mathrm{~cm}$ en el perfil del médano; (b) radio derecho de Puma concolor colectado en el muestreo del basural histórico; (c) asta de Hippocamelus bisulcus hallado en la margen Norte de la Laguna.

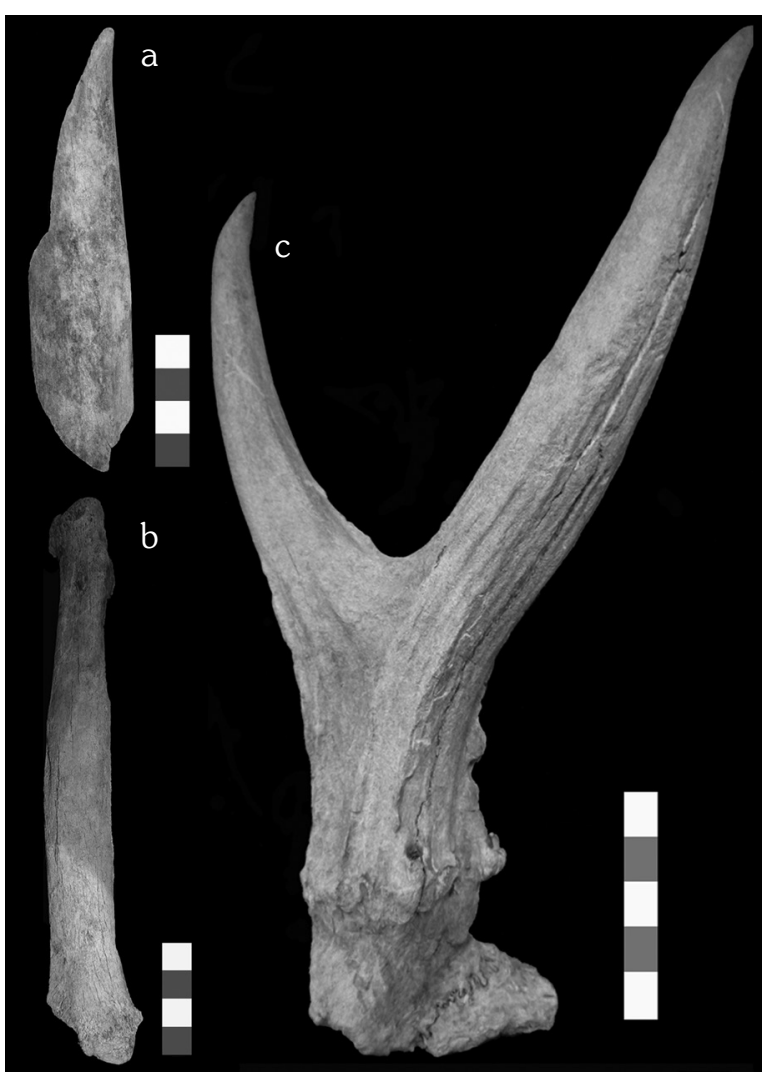

los estadios de fusión epifisiaria (Kaufmann, 2009), indican la presencia de 2 guanacos adultos ( $>36$ meses), 1 subadulto (24-36 meses) y 2 juveniles ( $<24$ meses). La representación de partes esqueletales de guanaco $(\mathrm{MNE}=40)$ muestra la prevalencia de elementos del esqueleto apendicular (huesos largos y del autopodio, 72,5 \%) y una moderada representación de elementos de la región axial (cráneo, costillas y vértebras, 27,5\%), indicando tanto una selección de partes y su transporte desde el lugar de matanza, como el ingreso de animales enteros al sitio para su explotación integral.

Por otro lado, se considera importante destacar la presencia de otros taxa identificados entre la fauna de LC. En el muestreo del sector del basural histórico se halló un radio completo semienterrado (Fig. 5b) y un fragmento de escápula de puma, ambos sin modificaciones atribuibles a la explotación antrópica. Se encontraron asociados tanto a material aborigen como europeo y su hallazgo en el conjunto faunístico de superficie fue interpretada como una incorporación natural. A diferencia, el caballo europeo identificado en los muestreos de superficie no fue asociada a un contexto moderno de acumulación, ya que existen numerosas referencias históricas que señalan la presencia de campamentos Aónikenk en la zona de los Morros (Childs, 1936; Gómez Otero, 1991; Martinic, 1995; Musters, 1964, entre otros). El uso de este espacio por cazadores Aónikenk a caballo podría explicar la asociación física de restos arqueológicos indígenas con especímenes de Equus caballus con modificaciones antrópicas hacia ca. 200 años AP.

Fig. 6. (a) Dominancia (Dominance) y homogeneidad (Evenness) de especies en Laguna Cóndor; (b) Densidades de hallazgos en los sondeos de Laguna Cóndor a

b
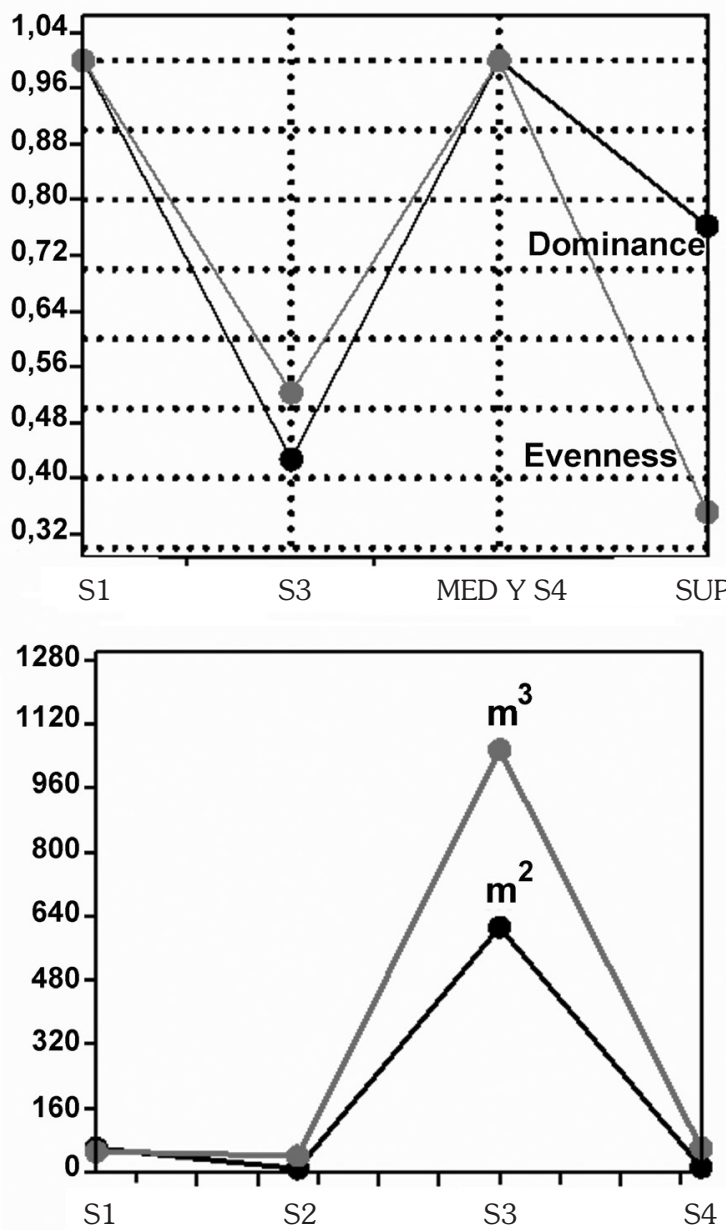
Se destaca la presencia de huemul en el sitio. En total son tres elementos, un asta (Fig. 5c) y dos especímenes del autopodio (MNI 1) encontrados en superficie a más de 300 m entre sí. La distancia entre éstos podría explicarse por transporte humano local, teniendo en cuenta lo llamativo del asta y el tráfico de pobladores de la estancia por la zona. Como antecedente, se conoce el hallazgo de un asta de huemul sin modificaciones antrópicas recolectada en la margen Norte de la Laguna Little Hill, aproximadamente a $22 \mathrm{~km}$ al sudeste de LC (Carballo Marina et al. 2008). La presencia de huellas sobre una de las falanges halladas en LC indicaría que el animal habría sido el producto de una explotación ocasional en el bosque o ecotono. Actualmente el bosque primario se halla a $15 \mathrm{~km}$ de LC y, durante el Holoceno tardío, su extensión habría sido más oriental (Moreno et al. 2009). La distancia desde el sitio al bosque entraría fácilmente dentro de los rangos de acción de caza Aónikenk, que contaban con la movilidad del caballo. Asimismo no puede descartarse su captura en el ecotono si se considera la información zooarqueológica y de su distribución actual que avala que este cérvido utilizaba tanto ambientes ecotonales como de bosque abierto (Fernández et al. 2015).

\section{CONSIDERACIONES FINALES}

En LC se observó una elevada diversidad de especies recuperadas aunque baja riqueza de taxa explotados. El análisis taxonómico expone una estrategia dirigida fundamentalmente al uso de mamíferos de estepa, guanaco, aunque también se registró la explotación de recursos de bosque, huemul y de fauna introducida, caballo.

La riqueza de especies identificadas fue mayor que la observada en sitios estudiados hacia el oeste en zona de bosque (Cancha Carrera 1) y al norte en ambientes de estepa (Cerro León 3 y 6 ; L'Heureux \& Borrazzo, 2013) y, en consonancia con la evidencia de tecnología lítica y de materias primas (Charlin et al. 2011; Charlin, 2012), se vincula con sitios estudiados hacia el oriente (e.g. OB1; L'Heureux, 2008).

En el área se observó una baja tasa de depositación ósea general con densidades puntuales elevadas (sondeo 3) y fragmentación elevada (sondeo 3), indicando ocupaciones puntuales e intensas en la Laguna Cóndor (Fig. 6b). Estos resultados y la evidencia proporcionada en relación con una explotación dirigida hacia grandes mamíferos de estepa, resulta concordante con la interpretación que la Laguna fue un espacio explotado logísticamente en forma recurrente pero durante períodos relativamente breves hacia ca. 200 años AP (Charlin et al. 2011; Charlin, 2012). Esta interpretación se aviene con la información suministrada por Hans Roehr quien menciona que, durante su estadía en Glencross, recuperó más de 40 bolas en proximidades de la Laguna Cóndor (F. Martin, com. pers.).

Si bien la muestra faunística estudiada en LC no es abundante, adquiere relevancia en tanto permite avanzar sobre el conocimiento acerca de la disponibilidad y el acceso a especies de bosque y estepa, como sobre la explotación que hicieron de la fauna los grupos de cazadores recolectores históricos que utilizaron este espacio y sus recursos durante los últimos siglos.

\section{AGRADECIMIENTOS}

A Arturo Menéndez y familia y a Matías Cedermas por su colaboración durante los trabajos de campo. A Fabiana Martin y Cecilia Pallo por los datos proporcionados vertidos en este trabajo. Al equipo de las campañas 2008 y 2011: Judith Charlin, Karen Borrazzo y Cecilia Pallo. A Sergio Lucero, División de Mastozoología, MACN.

\section{BIBLIOGRAFÍA}

Behrensmeyer, K. A. (1978). Taphonomic and ecological information from bone weathering. Paleobiology 41, 50-162.

Borrero, L. A., Charlin, J., Barberena, R., Martin, F. M., Borrazzo, K., \& L'Heureux, G. L. (2008). Circulación humana y modos de interacción al sur del río Santa Cruz. En L. A. Borrero \& N. V. Franco (Comps.), Arqueología del extremo sur del continente americano. Resultados de nuevos proyectos (pp. 155-174). Buenos Aires, Editorial Dunken.

Buscaglia, S. 2011. Informe del análisis de los materiales arqueológicos recuperados en Laguna Cóndor (Pcia. de Santa Cruz) campañas 2008-2011. MS.

Carballo Marina, F., Manzi, L., Campan, P., Belardi, J. B., Tiberi, P., Manera, A., \& Sáenz, J. L. (2008). 
Distribución del registro arqueológico en la cuenca del río Gallegos (Santa Cruz): línea de base y aporte a la preservación del patrimonio. En L. A. Borrero \& N. V. Franco (Comps.), Arqueología del extremo sur del continente americano. Resultados de nuevos proyectos, (pp. 175-225). Buenos Aires, Editorial Dunken.

Charlin, J., Borrero, L., \& Pallo, M. C. (2011). Ocupaciones humanas en el área noroccidental del río Gallegos (Prov. Santa Cruz, Argentina). En L. A. Borrero y K. Borrazzo (Eds.), Bosques, montañas y cazadores: biogeografía en Patagonia meridional (pp. 179-210). CONICET-IMHICIHU. Buenos Aires.

Charlin, J. (2012). Materias primas líticas y uso del espacio en las nacientes del río Gallegos: el caso de Laguna Cóndor (Estancia Glencross, Santa Cruz, Argentina). Magallania, 40(1), 163-184.

Childs, H. (1936). El Jimmy, A Patagonian Outlaw. FiladelfiaLondres, J. B. Lippincott Company.

Fernández, P. M., Cruz, I., Belardi, J. B., De Nigris, M., \& Muñoz, S. (2015). Human Predation and Natural History of Huemul(Cervidae; Hippocamelus bisulcus Molina, 1782) in Patagonia: a Zooarchaeological Analysis. Journal of Ethnobiology, 35(3), 472498.

Gómez Otero, J. (1991). Discusión sobre el límite occidental del territorio de los Proto-Tehuelches y Tehuelches meridionales en el extremo Sud de Patagonia (cuenca del río Gallegos). Waxen, 3, 5-21.

Gotelli, N., \& Graves, G. R. (1996). Null models in ecology. Cambridge, Smithsonian Institution Press.

Kaufmann, C. A. (2009). Estructura de Edad y Sexo en Lama guanicoe (Guanaco). Estudios actualísticos y arqueológicos en Pampa y Patagonia. Buenos Aires,
Sociedad Argentina de Antropología.

L'Heureux, G. L. (2008). La arqueofauna del Campo Volcánico Pali Aike. El sitio Orejas de Burro 1, Santa Cruz, Argentina. Magallania, 36(1), 65-76.

L'Heureux, G. L. \& Borrazzo, K. (2013). La arqueofauna del sudeste de sierra baguales durante el Holoceno: el sitio Cerro León 3 (Santa Cruz, Argentina). Magallania, 41(2), 223-240.

Lyman, R. (1994). Quantitative units and terminology in zooarchaeology. American Antiquity, 59, 36-71.

Martinic, M. (1995). Los Aónikenk. Historia y cultura. Punta Arenas, Ediciones Universidad de Magallanes.

Molinari, R. (2000). Relevamiento arqueológico en la estancia Rincón de los Morros (Santa Cruz). MS.

Musters, G. C. (1964 [1871]). Vida entre los Patagones. Un año de excursiones por tierras no frecuentadas desde el Estrecho de Magallanes hasta el río Negro. Buenos Aires, Solar/Hachette.

Moreno, P. I., Francois, J. P., Villa-Martinez, R., \& Moy, M. C. (2009). Millenial-scale variability in Southern Hemisphere westerly wind activity over the last 5000 years in SW Patagonia. Quaternary Science Reviews, 28, 25-38.

Ortiz Troncoso, O. (1973). Artefactos de silex de una tumba de morro Philippi, valle medio del río Gallegos (Prov. de Santa Cruz, Rep. Argentina). Anales del Instituto de la Patagonia, IV(1-3), 131-139.

Peri, P. L., Martínez Pastur, G., Monelos, L., \& Beroiz M. (2013). La distribución continental más oriental de Nothofagus antarctica en el río Gallegos (Santa Cruz). Anales Instituto Patagonia, 41(1),113-117.

Ramírez, C., Correa, M., Figueroa, H., \& San Martín, J. (1985). Variación del hábito y hábitat de Nothofagus antarctica en el sur de Chile. Bosque, 6(2), 55-73. 
